ARTICLE

https://doi.org/10.1038/s41467-019-10856-z

\title{
High efficiency perovskite quantum dot solar cells with charge separating heterostructure
}

\author{
Qian Zhao 1,2,3, Abhijit Hazarika (1) 2, Xihan Chen (10 2 ', Steve P. Harvey (1) 2, Bryon W. Larson (1) 2, \\ Glenn R. Teeter (1) 2, Jun Liu², Tao Song², Chuanxiao Xiao (1) 2, Liam Shaw4, Minghui Zhang', Guoran Li (1) ${ }^{3}$, \\ Matthew C. Beard ${ }^{2} \&$ Joseph M. Luther (iD ${ }^{2}$
}

Metal halide perovskite semiconductors possess outstanding characteristics for optoelectronic applications including but not limited to photovoltaics. Low-dimensional and nanostructured motifs impart added functionality which can be exploited further. Moreover, wider cation composition tunability and tunable surface ligand properties of colloidal quantum dot (QD) perovskites now enable unprecedented device architectures which differ from thin-film perovskites fabricated from solvated molecular precursors. Here, using layer-by-layer deposition of perovskite QDs, we demonstrate solar cells with abrupt compositional changes throughout the perovskite film. We utilize this ability to abruptly control composition to create an internal heterojunction that facilitates charge separation at the internal interface leading to improved photocarrier harvesting. We show how the photovoltaic performance depends upon the heterojunction position, as well as the composition of each component, and we describe an architecture that greatly improves the performance of perovskite QD photovoltaics.

\footnotetext{
${ }^{1}$ College of Chemistry, Nankai University, 300071 Tianjin, China. ${ }^{2}$ National Renewable Energy Laboratory, Golden, CO 80401, USA. ${ }^{3}$ Institute of New Energy Chemistry Material, Nankai University, 300350 Tianjin, China. ${ }^{4}$ Warren Wilson College, Asheville, NC 28815, USA. Correspondence and requests for materials should be addressed to J.M.L. (email: joey.luther@nrel.gov)
} 
$\mathrm{H}$ eterostructures within optoelectronic devices offer unique control of the electron and hole energy levels throughout the device ${ }^{1-3}$. For example, in photovoltaic (PV) devices, energy band engineering can enable better harvesting of photogenerated charge carriers ${ }^{4-6}$. This concept is especially prevalent in vapor deposited heterostructures using MOCVD and MBE of III-V semiconductors ${ }^{7}$ as well as in sputtered or thermally evaporated systems such as CIGS where the composition at surfaces can be altered to promote a buried location for the actual $p$ - $n$ charge separating junction ${ }^{8,9}$. In solution processed metal halide perovskite semiconductors, such a concept has not been successfully demonstrated primarily due to there being no existing pathway to prevent the solvation of the first deposited perovskite layer by the solvent of the subsequently deposited perovskite layer. In contrast, for perovskite QD films, the solvent does not technically dissolve the precursor materials, but rather suspends the QDs by their surface ligands in a colloidal solution. In layer-by-layer deposition of perovskite QD films, a layer of QDs is deposited from the colloidal solution and then ligands are chemically removed, rendering the resulting film insoluble to the nonpolar solvent, thus, enabling the ability to process the subsequent QD-layers without affecting the underlying film ${ }^{10-12}$. In our standard process, a QD-colloid in hexane or octane contains perovskite QDs terminated with organic ligands and is first deposited onto the desired substrate. The resulting QD film is treated with methyl acetate (MeOAc) that effectively removes native oleate ligands ${ }^{12}$. A second QD deposition can then be applied and the ligand removal process is repeated. In principle, the second (or any subsequent) solution can contain either the same QDs or QDs of different composition allowing for the composition of the perovskite film to be modulated in almost any desired order throughout the film thickness.

\section{Results}

Demonstration of heterojunction. To demonstrate the compositional heterojunction (schematically shown in Fig. 1a) as described above, we employ time-of-flight secondary ion mass spectrometry (ToF-SIMS) depth profiling ${ }^{13}$ on various films prepared herein (Fig. 1b-d). The SIMS detects signals for Cs, formamidinium (FA), $\mathrm{Pb}, \mathrm{I}, \mathrm{Ti}$, and In while sputtering through the film using an ion beam ${ }^{13}$. First, a pure $\mathrm{CsPbI}_{3}$ QD film on $\mathrm{TiO}_{2}$ is characterized and the $\mathrm{Cs}, \mathrm{Pb}$ and I signals of the QD film are observed in the ToF-SIMS depth profile. (Fig. 1b) At roughly $300 \mathrm{~nm}$ into the film, a sharp rise of the Ti signal becomes prominent indicating the ion beam has reached the underlaying $\mathrm{TiO}_{2}$ layer. In Fig. 1c, we show ToF-SIMS for a heterostructured film containing $\mathrm{CsPbI}_{3}$ QDs deposited on top of $\mathrm{Cs}_{0.25} \mathrm{FA}_{0.75} \mathrm{PbI}_{3} \mathrm{QDs}$. At roughly $200 \mathrm{~nm}$ into the film, the Cs signal decreases slightly while the FA signal rises (Fig. 1c). The distinct and sharp interface (rising several orders of magnitude) seen here with ToF-SIMS is nearly as sharp as the $\mathrm{CsPbI}_{3}$ interface with $\mathrm{TiO}_{2}$ in such a sample. In Fig. 1d, the perovskite compositions of the film structure are reversed compared to Fig. 1c, and here it is clear that a similar interface is formed within the film stack with the diminishment of the FA signal along with a slight increase in the Cs signal. This indicates a clear interface between layers of QDs with different compositions is formed and the sequence of these two QD layers can also be controlled via layer-by-layer deposition. In Supplementary Fig. 1 we show using ToF-SIMS that the integrity of such heterojunctions is minimally compromised after aging or moderate heating.

Optical and electronic properties. The successful construction of perovskite heterostructured films allows the investigation of the resulting composite optoelectronic properties. First, the electronic properties of the heterostructure are studied with X-ray photoelectron and ultraviolet photoelectron spectroscopies (XPS and UPS) to probe energy band positions in films of each QD composition (see Methods and Supplementary Fig. 2 for details and analysis). In Fig. 2a, we plot the resulting energy level positions for each QD film. The measured energy levels indicate that a higher FA content leads to deeper conduction band edges, valence-band edges, and Fermi level positions relative to vacuum. Thus, there is an opportunity to create an energy offset that could drive electrons towards a layer containing more FA while holes are driven toward layers with less FA. The band positions for spiro-OMeTAD, $\mathrm{TiO}_{2}$ and contacts are also shown in Fig. 2a for reference.

Next, we determine how the macroscopic optical properties of the films are controlled by the varying thickness and composition of each layer. It has been previously shown that perovskite QD devices with $~ 300$-nm-thick perovskite QD films have optimized power conversion efficiencies (PCEs) ${ }^{10,14,15}$. To be consistent, we perform a 4-cycle spin-coating procedure which, due to the QD concentration and solution properties, leads to a perovskite QD film with a total thickness of $\sim 300 \mathrm{~nm}$. Figure $2 \mathrm{~b}$ presents the external quantum efficiency (EQE) of PV devices as a function of the internal heterostructure interface position of the $\mathrm{Cs}_{0.25} \mathrm{FA}_{0.75} \mathrm{PbI}_{3}$ portion to that of $\mathrm{CsPbI}_{3}$. Two main features to note are the systematic shift in the absorption onset from 720 $\mathrm{nm}$ for pure $\mathrm{CsPbI}_{3}$ films to $790 \mathrm{~nm}$ for a film containing only $\mathrm{Cs}_{0.25} \mathrm{FA}_{0.75} \mathrm{PbI}_{3}$ and a reduction in the collection efficiency of blue wavelengths for the devices containing higher amounts of FA within the film. In addition, the effect of changing the composition of lower-bandgap bottom layer in EQE is also investigated and presented in Fig. 2c. The composition of the bottom layer again causes a systematic shift in the absorption onset of the composite film but is less dramatic than observed in Fig. 2b.

Device design and performance. Given the optical properties of the heterojunction-containing films and the energetics shown above, Fig. 3 presents PV performance of the corresponding devices. Using the device architecture shown in Fig. 3a, a series of solar cells were fabricated (EDS elemental mapping of the cross section and morphology of QDs presented in Supplementary Figs. 3 and 4 , respectively). Figure $3 \mathrm{~b}$ shows $J-V$ curves for devices to investigate the impact of thickness ratio of $\mathrm{Cs}_{0.25} \mathrm{FA}_{0.75} \mathrm{PbI}_{3}$ layer to $\mathrm{CsPbI}_{3}$ layer in the perovskite QD absorber. However, since we observe non-negligible hysteresis during the reverse and forward scans of devices (shown in Supplementary Fig. 5), the power conversion efficiency (PCE) from stabilized power output (SPO) at $0.950 \mathrm{~V}$ is reported (Fig. 3c). This figure of merit (PCE from SPO) has now become required for accurate reporting of efficiency for perovskite solar cells ${ }^{16,17}$. The reproducibility of these devices is demonstrated by showing the histograms of PCE from SPO (Supplementary Fig. 6). Despite similar PCE from the reverse scans for several devices, the device containing layer ratio of $1: 3\left(\mathrm{Cs}_{0.25} \mathrm{FA}_{0.75} \mathrm{PbI}_{3}: \mathrm{CsPbI}_{3}\right)$ has the highest PCE of $15.52 \%$ (see Table 1 and parameters from the forward scans presented in Supplementary Table 1 as well as heterojunction layer and device stability in the Supplementary Figs. 1 and 7). The improvement is mainly attributed to increased $J_{\mathrm{sc}}$ and suggests more photogenerated charge carriers are harvested with this architecture ${ }^{18}$. As a control experiment, devices from simply blending the two QD solutions at the same ratio (1:3 $\mathrm{Cs}_{0.25} \mathrm{FA}_{0.75} \mathrm{PbI}_{3}: \mathrm{CsPbI}_{3}$ ) were also prepared (Supplementary Fig. 8). It is anticipated that this would form more of a bulk heterojunction architecture rather than the planar heterojunction films described here. These bulk heterojunction QD devices show 
a

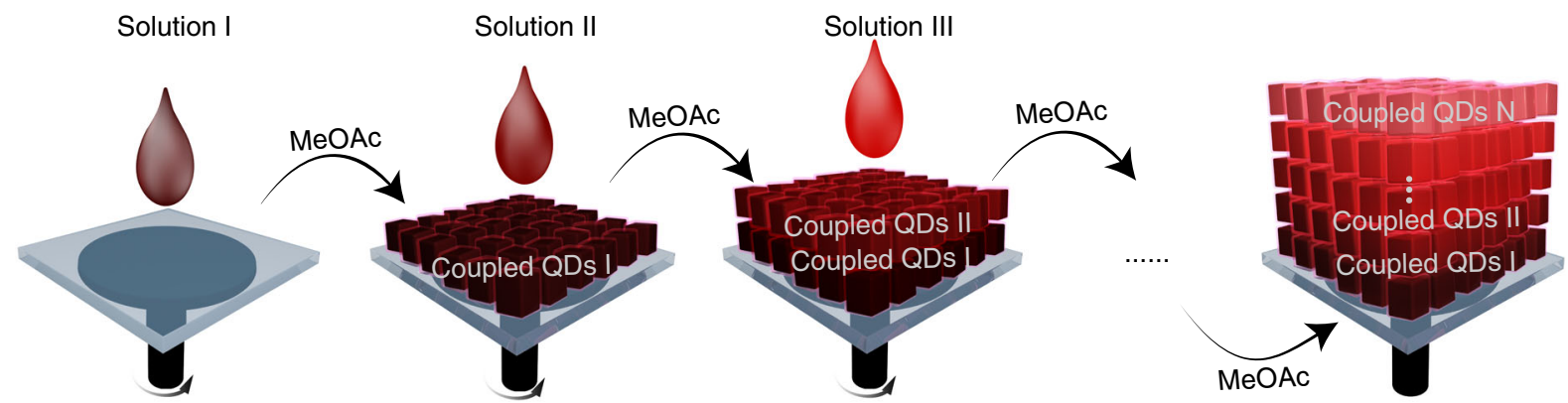

b

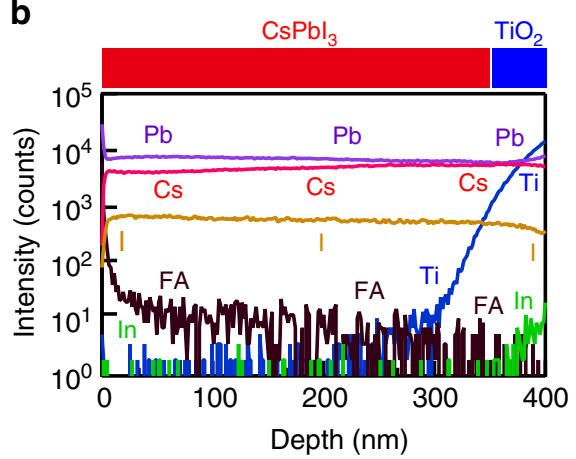

C

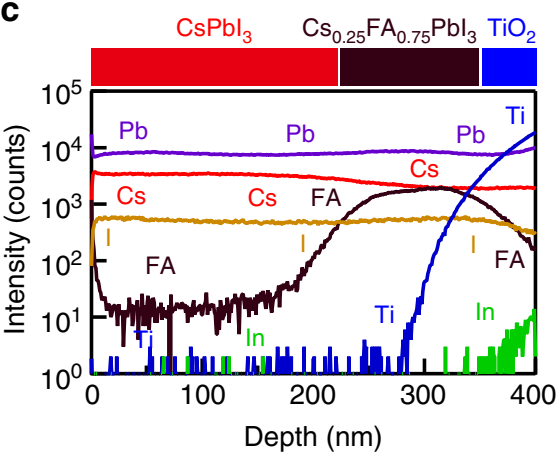

d

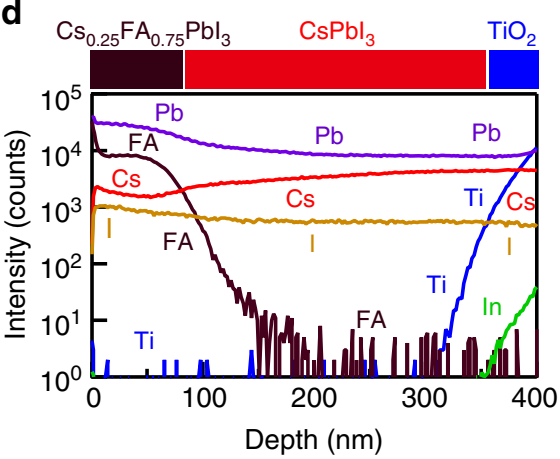

Fig. 1 Heterojunction structure of QD films. a Schematic overview of layer-by-layer assembly showing a perovskite QD film composed of different layers of QDs. MeOAc treatment is carried out between the deposition of different QD layers to remove the native oleate ligands and to render the deposited QDs insoluble in the solvent. b-d ToF-SIMS depth profile of samples with the structure and interface location indicated in the bar above the plots of $\mathrm{CsPb}_{3} / \mathrm{TiO}_{2}$ (b), $\mathrm{CsPbl}_{3} / \mathrm{Cs}_{0.25} \mathrm{FA}_{0.75} \mathrm{Pbl}_{3} / \mathrm{TiO}_{2}$ (c), and $\mathrm{Cs}_{0.25} \mathrm{FA}_{0.75} \mathrm{Pbl}_{3} / \mathrm{CsPb}_{3} / \mathrm{TiO}_{2}$ (d). All relevant elements from perovskite QD layer and $\mathrm{TiO}_{2}$ are shown. Compared to the structure without $\mathrm{Cs}_{0.25} \mathrm{FA}_{0.75} \mathrm{Pbl}_{3}$, the $\mathrm{FA}$ signal shows a sharp and significant change and the $\mathrm{Cs}$ signal also shows a slight change at the boundary between the $\mathrm{CsPb}_{3}$ and $\mathrm{Cs}_{0.25} \mathrm{FA}_{0.75} \mathrm{Pbl}_{3} \mathrm{QD}$ layers. The In signal is shown as a reference for when the ToF-SIMS profiling beam reaches the ITO on the substrate

characteristics similar to single component devices rather than the improved carrier harvesting seen in planar heterojunction devices.

Time-resolved carrier dynamics of heterojunction films. To rationalize the high PCE of perovskite/perovskite QD heterojunctions, the mobility and lifetime of photogenerated carriers in each QD film were obtained using time-resolved microwave conductivity (TRMC). We find the carriers in the more FAcontaining films possess longer free-carrier lifetimes but also exhibit lower effective mobility compared to pure $\mathrm{CsPbI}_{3}$ QD films (Supplementary Fig. 9). In other words, the trade-off in lower carrier mobility for longer average carrier lifetime results in increased recombination and less charge collection for thick FAcontaining layers. Consequently, we observe a decrease in $F F$ and $J_{\mathrm{sc}}$ for the devices with excessively thick FA-containing layers. This also helps to explain the reduction of EQE shown for 350 to $550 \mathrm{~nm}$ wavelengths in Fig. 2b. Light with wavelength around $450 \mathrm{~nm}$ is primarily absorbed in the FA-containing heterojunction component and shows poorer collection in thicker FAcontaining layers. In Fig. $3 \mathrm{~d}$, e, we present $J-V$ curves and SPO for devices with different compositions of the lower bandgap layer in the heterojunction with the optimized heterojunction position determined from Fig. 3c. There is negligible difference for device performance when the bottom-most layer of the absorber is $\mathrm{Cs}_{0.75} \mathrm{FA}_{0.25} \mathrm{PbI}_{3}, \mathrm{Cs}_{0.5} \mathrm{FA}_{0.5} \mathrm{PbI}_{3}, \mathrm{Cs}_{0.25} \mathrm{FA}_{0.75} \mathrm{PbI}_{3}$, or $\mathrm{FAPbI}_{3}$ (see Supplementary Table 2), which may be associated with the slightly different energy levels and that the fast charge separation is similar in each case.

To better understand the charge separation dynamics induced by the designed heterojunction, we performed transient absorption (TA) spectroscopy which can independently probe carrier populations in each component of the heterojunction upon illumination. In the TA spectra (presented as pseudocolor images, Fig. 4) near the bandgap energy, a reduced absorption (ground state bleach) generally corresponds to the presence of excitons or free-charge carriers and the center of the reduced absorption indicates the exciton transition energy ${ }^{19}$. In Fig. 4 we show TA data for a heterojunction film excited at $400 \mathrm{~nm}$ either through the high bandgap $\mathrm{CsPbI}_{3}$ side (Fig. 4a) or through the lower bandgap $\mathrm{Cs}_{0.25} \mathrm{FA}_{0.75} \mathrm{PbI}_{3}$ side (Fig. 4c) as well as single component films of each composition (Fig. 4f, g). The anticipated energy band diagram is shown in Fig. 4 b, indicating a $180-\mathrm{meV}$ conduction band offset that will drive electrons towards the FAcontaining component. The valence-band offset favors hole transfer to $\mathrm{CsPbI}_{3}$ but has only a small energetic offset of 20 $\mathrm{meV}$, which is less than $k T$ at room temperature, thus holes will mainly diffuse in the heterostructure. The TA spectra cannot be represented by only one bleaching component and we model our data by a sum of two Gaussian peaks, one centered at $697 \mathrm{~nm}$ and corresponds to carriers residing in the $\mathrm{CsPbI}_{3}$ component and another centered at $728 \mathrm{~nm}$ corresponding to carriers residing in the $\mathrm{Cs}_{0.25} \mathrm{FA}_{0.75} \mathrm{PbI}_{3}$ component. In Fig. $4 \mathrm{a}$, a strong bleach is mainly observed at $697 \mathrm{~nm}$ due to excitation into the $\mathrm{CsPbI}_{3}$ component. As the delay between the pump and probe beam is swept between 1 ps and 2000 ps, the bleach component at $697 \mathrm{~nm}$ is transferred to one at $728 \mathrm{~nm}$ due to electron transfer from $\mathrm{CsPbI}_{3}$ to $\mathrm{Cs}_{0.25} \mathrm{FA}_{0.75} \mathrm{PbI}_{3}$. In Fig. $4 \mathrm{~d}$, the fractional component of the bleach in each component is plotted versus delay time and we find that initially $80 \%$ of the bleach is composed of that attributed to the $\mathrm{CsPbI}_{3}$ while $\sim 20 \%$ initially penetrates through the $\mathrm{CsPbI}_{3}$ to $\mathrm{Cs}_{0.25} \mathrm{FA}_{0.75} \mathrm{PbI}_{3}$. The fractional component in the 

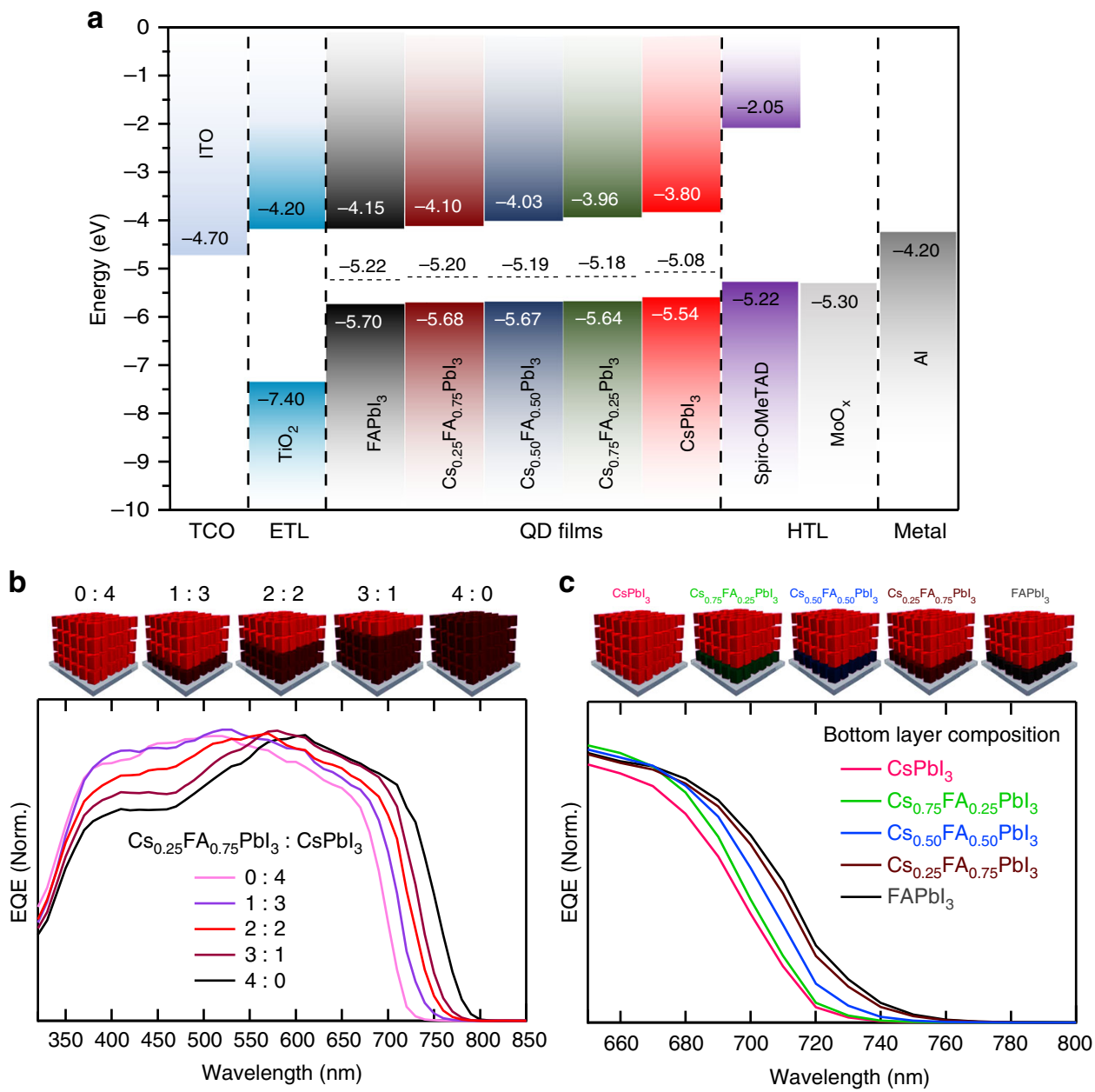

Fig. 2 Optical and electronic properties of perovskite QD heterojunction. a Energy band positions for perovskite QD compositions and contact layers considered in this work. The Fermi level positions (denoted by horizontal dotted lines) and valence-band edges $\left(E_{\mathrm{v}}\right)$ of all perovskite QD films were determined by UPS and XPS, respectively. The conduction band edges $\left(E_{\mathrm{c}}\right)$ were calculated by adding the bandgap energy ${ }^{26}$ to $E_{\mathrm{v}}$ for the corresponding perovskite QD films. $\mathbf{b}$ EQE spectra of solar cells with varying thickness ratio of the $\mathrm{Cs}_{0.25} \mathrm{FA}_{0.75} \mathrm{Pbl}_{3}$ layer to $\mathrm{CsPb}_{3}$ layer in the perovskite $\mathrm{QD}$ absorber. c EQE spectra of solar cells with different compositions of the bottom layer in the perovskite QD absorber where the thickness ratio of the mixed-cation $\mathrm{Cs}_{x} \mathrm{FA}_{1-\mathrm{x}} \mathrm{Pbl}_{3} \mathrm{QD}$ layers to $\mathrm{CsPb}_{3} \mathrm{QD}$ layers is 1:3 in all cases

$\mathrm{CsPbI}_{3}$ decreases with time with a characteristic electron transfer time of $\sim 600 \mathrm{ps}$. Such induced charge separation is advantageous for suppressing recombination and enhancing charge extraction ${ }^{20,21}$.

In Fig. $4 \mathrm{e}$, the fractional components are plotted when the film is instead excited through the $\mathrm{Cs}_{0.25} \mathrm{FA}_{0.75} \mathrm{PbI}_{3}$ side. Again, about $20 \%$ of the excitation is transmitted through the $\mathrm{Cs}_{0.25} \mathrm{FA}_{0.75} \mathrm{PbI}_{3}$ to the $\mathrm{CsPbI}_{3}$ back layer in this case and the bleach signal remains roughly constant with time. In the lower panel in Fig. 4a, c, selected line cuts from the surface plots are shown along with component Gaussian fits to better visualize the charge transfer. The selected cuts in Fig. 4a show the increasing component of the bleach growing in at 153 ps and 1865 ps relative to 1.48 ps after excitation. In Fig. 4c, initially at 1.48 ps, a small $20 \%$ component of the bleach is due to the $\mathrm{CsPbI}_{3}$ layer as discussed but this signal decreases with increased pump-probe delay since TA has been shown to be more sensitive to electron than hole signatures ${ }^{22}$ and a much lesser driving force for hole transfer as indicated in the band diagram.

We also performed TA for pure single component $\mathrm{CsPbI}_{3} \mathrm{QD}$ and pure $\mathrm{Cs}_{0.25} \mathrm{FA}_{0.75} \mathrm{PbI}_{3}$ (shown in Fig. 4f, g, respectively). These single component films show a bleach at $688 \mathrm{~nm}$ for $\mathrm{CsPbI}_{3}$
QDs and $738 \mathrm{~nm}$ for the $\mathrm{Cs}_{0.25} \mathrm{FA}_{0.75} \mathrm{PbI}_{3}$ sample which do not evolve much with time. These spectra are provided for reference. As explained by this TA analysis, charge transfer inside the absorber induced by heterojunction efficiently separates photogenerated electrons and (to a lesser degree) holes driving them opposite directions.

\section{Discussion}

We develop perovskite devices that enables the rational design of charge separating interfaces within a perovskite QD absorber layer. By controlling the QD composition during layer-by-layer QD-film deposition, we can locate a spatial heterostructure arbitrarily within the resulting perovskite layer. We show how the composition of each heterostructure-component can be optimized based on charge-carrier lifetimes and mobilities within each component-QD to achieve a PCE from SPO up to $15.74 \%$. Our concept could be easily employed with QDs and other forms of absorber layers (thin films $s^{15}, 2 \mathrm{D}$ structures ${ }^{23}$, or combinations therein $^{24}$ ). Furthermore, our approach should lead to a larger design-space which will impact a wider range of perovskite-based optoelectronic applications such as LEDs, transistor, (spintronic or quantum information processing, etc.). 
a

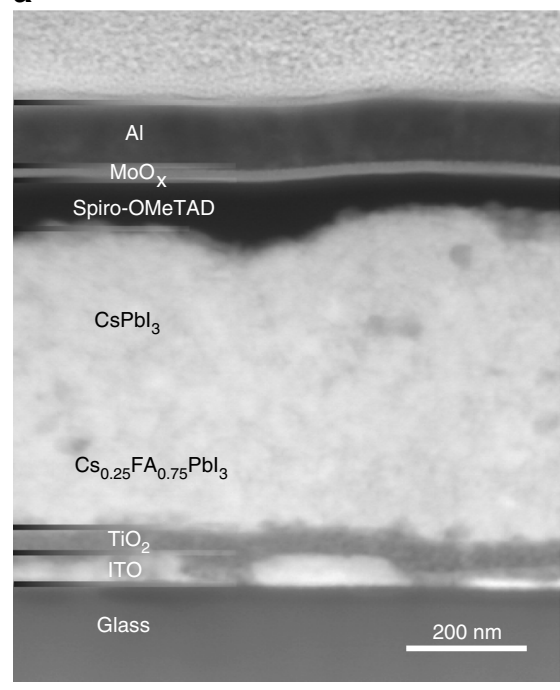

b

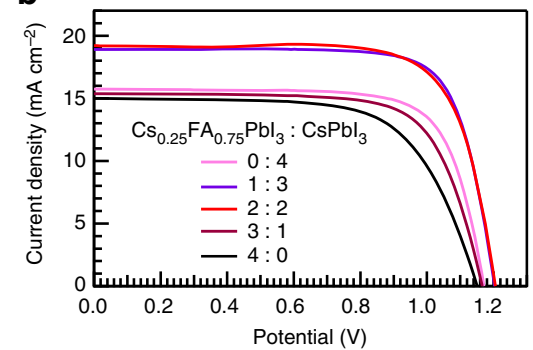

d

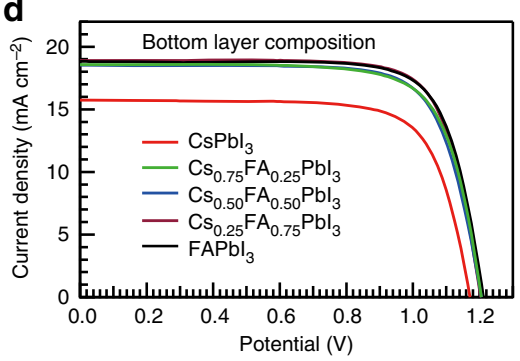

C

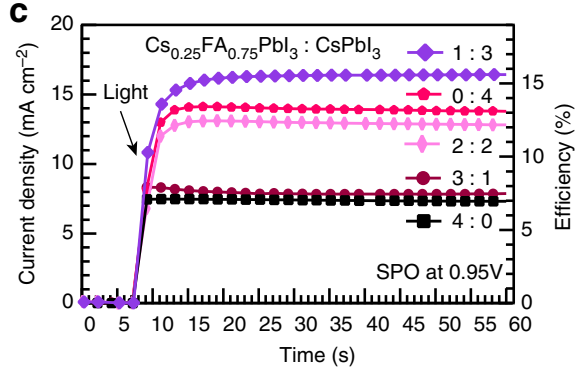

e

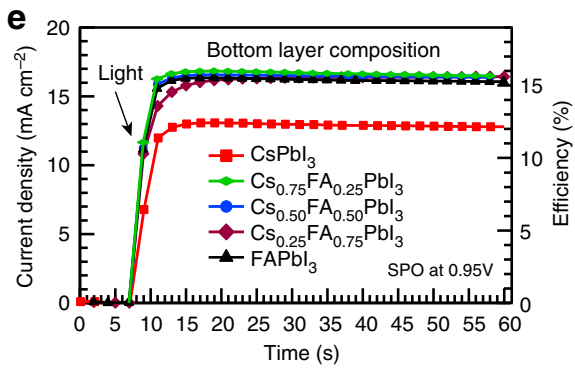

Fig. 3 Photovoltaic performance of solar cells. a Cross-sectional STEM-HAADF image of devices with the structure of $\mathrm{Glass}_{\mathrm{ITO} / \mathrm{TiO}} / \mathrm{Cs}_{0.25} \mathrm{FA}_{0.75} \mathrm{Pbl}_{3} /$ $\mathrm{CsPb}_{3} /$ spiro-OMeTAD/MoO $/ \mathrm{Al}$. b, c $J-V$ curves recorded by reverse scans (from open circuit to short circuit) and SPO at $0.95 \mathrm{~V}$ of devices with different thickness ratio of $\mathrm{Cs}_{0.25} \mathrm{FA}_{0.75} \mathrm{Pbl}_{3}$ layer to $\mathrm{CsPbl}_{3}$ layer in the perovskite $\mathrm{QD}$ absorber, respectively. $\mathbf{d}, \mathbf{e} J-V$ curves measured via reverse scans and SPO at $0.95 \mathrm{~V}$ of devices with different compositions of the bottom layer in the perovskite QD absorber where the heterojunction position is constant

Table 1 Photovoltaic parameters of solar cells

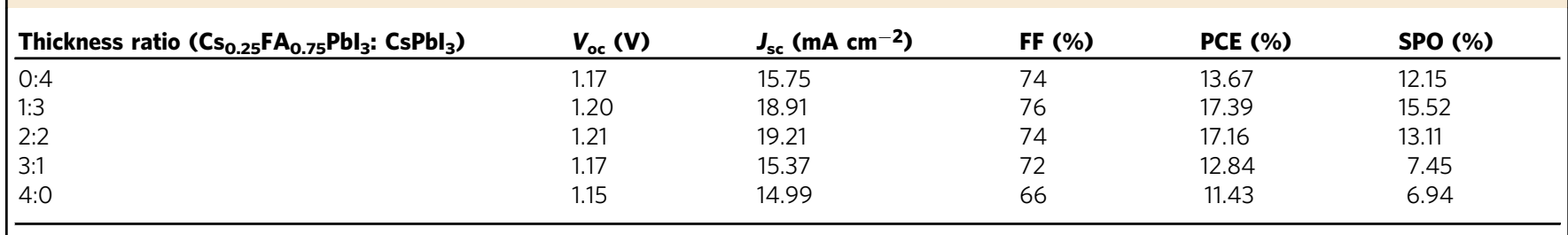

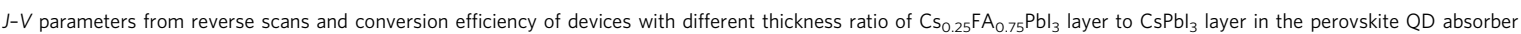

\section{Methods}

Materials. Formamidinium iodide $\left(\mathrm{CH}\left(\mathrm{NH}_{2}\right)_{2}, \mathrm{FAI}\right)$ was purchased from Dyesol. Lead (II) iodide $\left(\mathrm{PbI}_{2} ; 99.9985 \%\right)$ and bis(trifluoromethane)sulfonimide lithium salt (Li-TFSI) were purchased from Alfa Aesar. 2,2',7,7'-tetrakis(N,N-di-p-methoxyphenylamino)-9, $9^{\prime}$-spirobifluorene (spiro-OMeTAD; $\geq 99.5 \%$ ) was purchased from Lumtec. Cesium carbonate $\left(\mathrm{Cs}_{2} \mathrm{CO}_{3} ; 99.9 \%\right)$, oleylamine (OAm; technical grade, $70 \%$ ), oleic acid (OA; technical grade, $90 \%$ ), 1-octadecene (ODE; technical grade, $90 \%$ ), octane (anhydrous, $\geq 99 \%$ ), hexane (reagent grade, $\geq 95 \%$ ), methyl acetate (MeOAc; anhydrous, $99.5 \%)$, lead nitrate $\left(\mathrm{Pb}\left(\mathrm{NO}_{3}\right)_{2} ; 99.999 \%\right)$, ethyl acetate (EtOAc; anhydrous, 99.8\%), formamidinium acetate (FA-acetate, 99\%), titanium ethoxide ( $\geq 97 \%)$, hydrochloric acid ( $\mathrm{HCl} ; 37 \%$ in water), chlorobenzene (anhydrous, 99.8\%), 4-tert-butylpyridine (4-TBP; 96\%), toluene (anhydrous, 99.8\%), dimethylformamide (DMF), dimethyl sulfoxide (DMSO), and acetonitrile (anhydrous, 99.8\%) were purchased from Sigma-Aldrich and used as received unless otherwise specified.

$\mathrm{CsPb}_{3}$ QDs synthesis and purification. The synthesis was performed following the method reported in our previous publications with slight modification ${ }^{11,25}$. First, $0.407 \mathrm{~g}$ of $\mathrm{Cs}_{2} \mathrm{CO}_{3}$ was added in $20 \mathrm{~mL}$ of ODE containing $1.25 \mathrm{~mL}$ of OA and degassed at $150{ }^{\circ} \mathrm{C}$ for $20 \mathrm{~min}$ under vacuum in a $100-\mathrm{mL}$ three-neck flask. After $\mathrm{Cs}_{2} \mathrm{CO}_{3}$ was absolutely dissolved in the solution, the Cs-oleate precursor was completed and kept in $\mathrm{N}_{2}$ at $150^{\circ} \mathrm{C}$ until needed. Subsequently, $0.5 \mathrm{~g}$ of $\mathrm{PbI}_{2}$ and $25 \mathrm{~mL}$ of ODE were mixed in a $100-\mathrm{mL}$ three-neck flask at $120^{\circ} \mathrm{C}$ for $10 \mathrm{~min}$ under vacuum. A preheated mixture of $\mathrm{OA}$ and $\mathrm{OAm}\left(130^{\circ} \mathrm{C}, 2.5 \mathrm{~mL}\right.$ each) was injected into the $\mathrm{PbI}_{2}$ solution and the reaction flask was kept at $120^{\circ} \mathrm{C}$ until a clear solution was formed. Then the reaction flask was heated to $185^{\circ} \mathrm{C}$ under flowing $\mathrm{N}_{2}$. Once $2 \mathrm{~mL}$ of the Cs-oleate precursor was immediately injected into the reaction flask, the mixture was quenched in an ice bath. After cooling to room temperature, the colloidal solution was mixed with 70 to $80 \mathrm{~mL}$ of $\mathrm{MeOAc}$ and then centrifuged at $7500 \mathrm{rpm}$ for $5 \mathrm{~min}$. The precipitated QDs were dispersed in $5 \mathrm{~mL}$ of hexane and re-precipitated by adding about $5 \mathrm{~mL}$ of MeOAc. After centrifugation (7500 rpm, 5 $\mathrm{min}$ ), the resulting precipitate was redispersed in $15 \mathrm{~mL}$ of hexane and stored in refrigerator at $4{ }^{\circ} \mathrm{C}$. To remove excess Cs-oleate and $\mathrm{Pb}$-oleate, the $\mathrm{QD}$ solution was centrifuged at $7500 \mathrm{rpm}$ for $5 \mathrm{~min}$ after 24 hours of storage in refrigerator. The supernatant was filtered through a $0.45-\mu \mathrm{m}$ nylon filter and ready for used.

$\mathrm{FAPbl}_{3}$ QDs synthesis and purification. $\mathrm{FAPbI}_{3}$ QDs was synthesized and purified following the previously reported method ${ }^{26}$. The FA-oleate precursor was prepared by first degassing a mixture of $0.521 \mathrm{~g}$ of FA-acetate and $10 \mathrm{~mL}$ of OA in a $100-\mathrm{mL}$ three-neck flask under a vacuum at $120^{\circ} \mathrm{C}$ for $20 \mathrm{~min}$. Then $\mathrm{N}_{2}$ was introduced in the reaction flask and the clear reaction solution was yielded. After cooling to $90^{\circ} \mathrm{C}$, the FA-oleate precursor was kept in $\mathrm{N}_{2}$ and ready for injection. To prepare $\mathrm{PbI}_{2}$ solution, $0.344 \mathrm{~g}$ of $\mathrm{PbI}_{2}$ was added into $20 \mathrm{~mL}$ of ODE and degassed at $120^{\circ} \mathrm{C}$ for $20 \mathrm{~min}$ under vacuum in a $100-\mathrm{mL}$ three-neck flask. A preheated mixture of $\mathrm{OA}$ and $\mathrm{OAm}\left(130^{\circ} \mathrm{C}, 3 \mathrm{~mL}\right.$ each) was injected into the $\mathrm{PbI}_{2}$ solution and the reaction flask was kept at $120^{\circ} \mathrm{C}$ until a clear solution was formed. Purging the flask with $\mathrm{N}_{2}$, the $\mathrm{PbI}_{2}$ solution was cooled down and kept at $80^{\circ} \mathrm{C}$. FA-oleate precursor $(5 \mathrm{~mL})$ was immediately injected into the $\mathrm{PbI}_{2}$ solution. The mixture was quenched in an ice bath after $15 \mathrm{~s}$. MeOAc $(9 \mathrm{~mL})$ was added into the colloidal solution after cooling to room temperature and then centrifuged at $8000 \mathrm{rpm}$ for $30 \mathrm{~min}$. The precipitated QDs was dispersed in $9 \mathrm{~mL}$ of toluene. Around $10 \mathrm{~mL}$ of $\mathrm{MeOAc}$ was added into the QDs solution and then it was centrifuged at $8000 \mathrm{rpm}$ for $10 \mathrm{~min}$. The resulting precipitate was redispersed in $10 \mathrm{~mL}$ of octane and stored in refrigerator at $4{ }^{\circ} \mathrm{C}$ for at least $24 \mathrm{~h}$. Before use, the QDs solution was centrifuged at $7500 \mathrm{rpm}$ for $5 \mathrm{~min}$ and the precipitate was decarded. The supernatant was filtered through a $0.45-\mu \mathrm{m}$ nylon filter.

Preparation of $\mathbf{C s}_{\mathbf{1}} \mathbf{F A}_{\mathbf{x}} \mathbf{P b l}_{\mathbf{3}}$ alloys. Colloidal solutions of $\mathrm{CsPb}_{3}$ and $\mathrm{FAPbI}_{3}$ QDs were dispersed in octane, respectively. To obtain similar concentrations of these two solutions, the absorption spectra were measured, and each solution had a similar optical density near the band edge. After the concentrations were calibrated, these two colloidal solutions were mixed in different volume ratios to yield $\mathrm{Cs}_{1}$ ${ }_{x} \mathrm{FA}_{x} \mathrm{PbI}_{3}$ QDs with the desired Cs/FA stoichiometry and then stored at room temperature for at least $48 \mathrm{~h}^{26}$. 
a

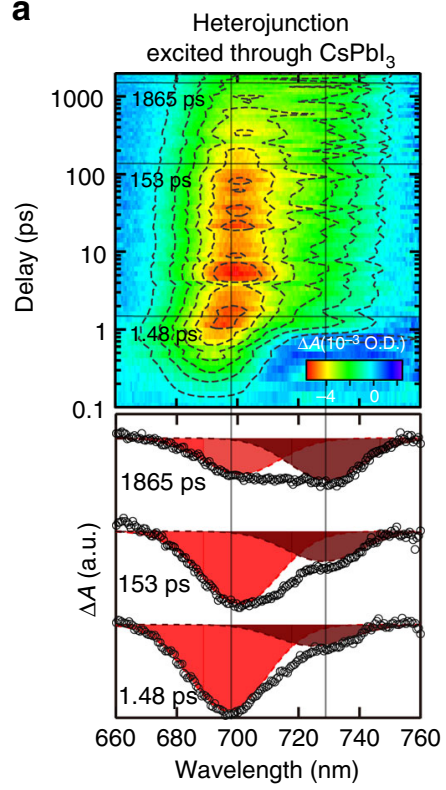

$\mathbf{f}$

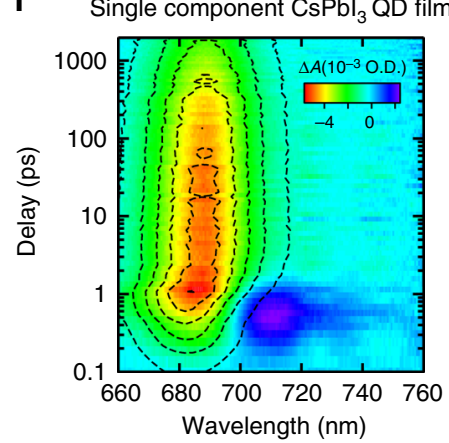

b
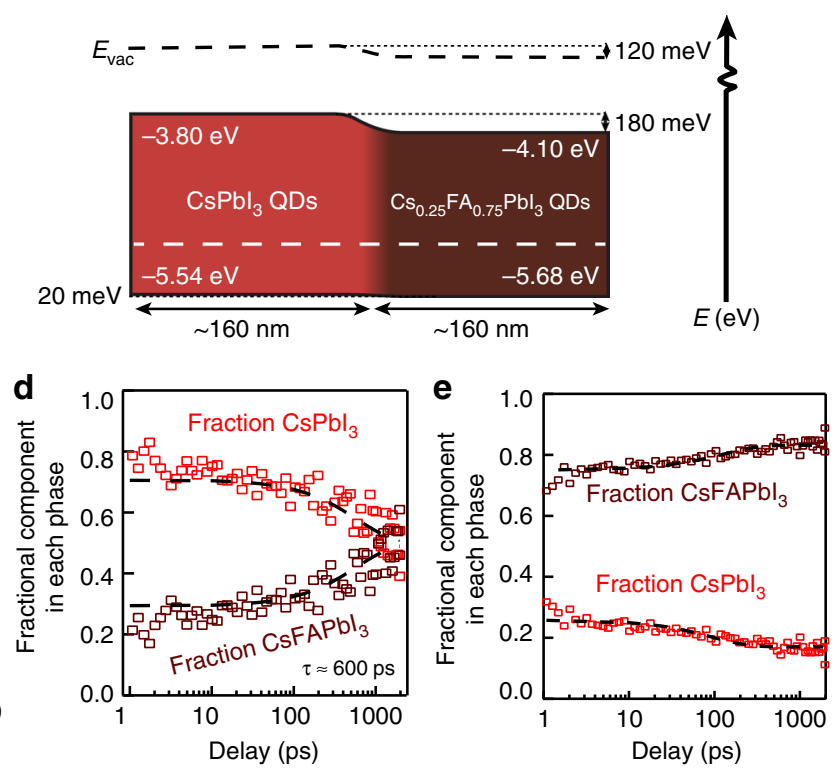

C

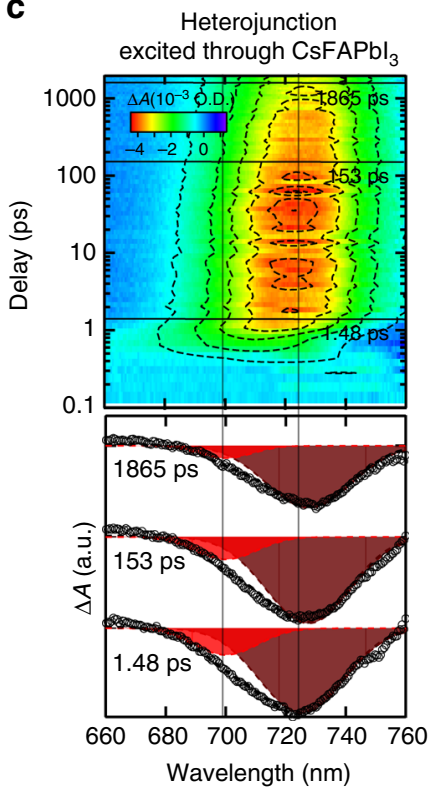

\section{g}

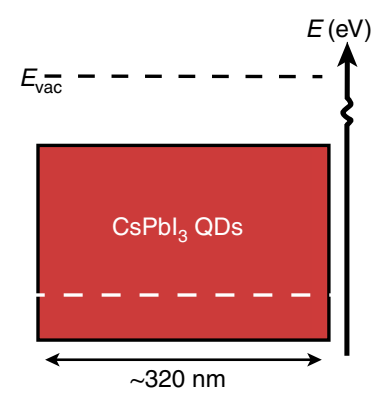

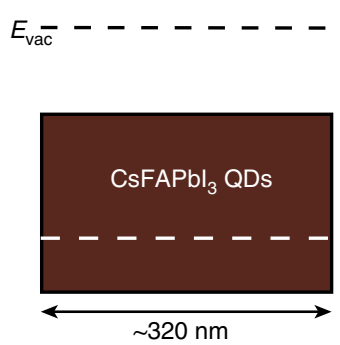

Single component $\mathrm{CsFAPbl}_{3} \mathrm{QD}$ film

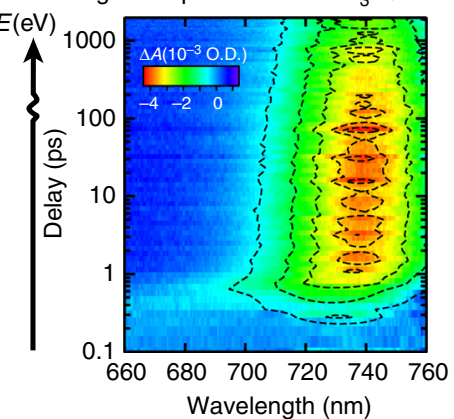

Fig. 4 Transient absorption of heterojunction QD films. a Pseudocolor image of transient response for QD heterostructure when photoexciting through the $\mathrm{CsPb}_{3}$ layer. For each of the surface plots with dashed contour lines one can visualize the carrier populations at various delay time delays between the pump and probe beam with the model described. Select cuts through the data are presented below the surface plots and offset vertically for clarity. Cuts are shown at 1.48 ps, 153 ps, and 1865 ps time delay. The spectra at each delay can be represented by the bleaching of two Gaussian peaks, one has a center wavelength at $697 \mathrm{~nm}$ (red-shaded Gaussian) and is associated with carriers occupying $\mathrm{CsPbl}_{3}$ while the other peak has a center wavelength at 728 $\mathrm{nm}$ (brown-shaded Gaussian) and is associated with carriers spatially occupying the $\mathrm{Cs}_{0.25} \mathrm{Fa}_{0.75} \mathrm{Pbl}_{3}$ component. Vertical lines show the position of the two centered components. b Energy level diagram of the heterostructured film with estimated band alignment from UPS. In experiment a light impinges from the left $\left(\mathrm{CsPbl}_{3} \mathrm{QDs}\right)$ while for $\mathbf{c}$ light impinges from the right $\left(\mathrm{Cs}_{0.25} \mathrm{FA}_{0.75} \mathrm{Pbl}_{3} \mathrm{QDs}\right)$. From the alignment, electrons are driven to the $\mathrm{FA}$-containing side with a conduction band offset of $180 \mathrm{meV}$ while holes have a small (20 meV) offset driving holes toward $\mathrm{CsPb}_{3} \mathbf{c} \mathrm{Pseudocolor}_{\mathrm{mage}}$ of transient response for QD heterostructure when photoexciting through the $\mathrm{Cs}_{0.25} \mathrm{FA}_{0.75} \mathrm{Pbl}_{3}$ layer. The coloring scheme of the decomposed spectra is the same as in a. $\mathbf{d}$, e The decomposed fraction of the TA signal for each delay measured that arises from the $\mathrm{CsPbl}_{3}$ (red-squares) and $\mathrm{Cs}_{0.25} \mathrm{FA}_{0.75} \mathrm{Pbl}_{3}$ (brown-squares) as a function of time when photoexciting through $\mathrm{CsPb}_{3}(\mathbf{d})$ or through $\mathrm{Cs}_{0.25} \mathrm{FA}_{0.75} \mathrm{Pbl}_{3}(\mathbf{e})$. For $\mathbf{d}$, the fraction of $\mathrm{CsPbl}_{3}$ decreases over time while $\mathrm{Cs}_{0.25} \mathrm{FA}_{0.75} \mathrm{Pbl}_{3}$ increases as electrons are transferred into the FA-containing layer with a charge transfer time average of around 600 ps. For $\mathbf{e}$, the fraction of $\mathrm{CSPbl}_{3}$ and $\mathrm{Cs}_{0.25} \mathrm{FA}_{0.75} \mathrm{Pbl}_{3}$ is analyzed after the first $\mathrm{ps}$ and then stay relatively unchanged. $\mathbf{f}$ Pseudocolor image (left) and diagram (right) of a thick single component $\mathrm{CsPb}_{3}$ film $\mathbf{g}$ Pseudocolor image (right) and diagram (left) of pure $\mathrm{Cs}_{0.25} \mathrm{FA}_{0.75} \mathrm{Pbl}_{3}$

Fabrication of QD films. Coupled $\mathrm{Cs}_{1-x} \mathrm{FA}_{x} \mathrm{PbI}_{3}$ QD films (where $x=0,0.25,0.50$, 0.75 , or 1.0 ) were deposited following previously reported methods ${ }^{26}$ with slight modifications. Deposition of all QD films was carried out in a humidity-controlled box (relative humidity, 20-25\%). First, saturated $\mathrm{Pb}\left(\mathrm{NO}_{3}\right)_{2}$ in $\mathrm{MeOAc}$ solution and FAI in EtOAc solution were prepared. Each layer of $\mathrm{Cs}_{1-x} \mathrm{FA}_{x} \mathrm{PbI}_{3} \mathrm{QD}$ was deposited by spin-coating at $1000 \mathrm{rpm}$ for $20 \mathrm{~s}$ and $2000 \mathrm{rpm}$ for $5 \mathrm{~s}$. Subsequently, the film was swiftly soaked into the $\mathrm{Pb}\left(\mathrm{NO}_{3}\right)_{2}$ solution and then rinsed in the neat $\mathrm{MeOAc}$ solution. A total film with $\sim 300 \mathrm{~nm}$ of thickness was obtained by repeating the process of spin-coating QDs and soaking in the solution four times. To achieve a heterojunction structure, $\mathrm{Cs}_{1-x} \mathrm{FA}_{x} \mathrm{PbI}_{3}$ QDs with different Cs/FA stoichiometry was deposited by following different sequences. Finally, the film was dipped in FAI solution for $10 \mathrm{~s}$ and rinsed in the neat $\mathrm{MeOAc}$ solution ${ }^{10,12}$.

Fabrication of PV devices. All of PV devices was fabricated by following previous literatures with slight modification ${ }^{10,11}$. Patterned indium-doped tin oxide
(ITO) glass was cleaned by sonicating in isopropanol for $30 \mathrm{~min}$ and treated with UV-ozone for $5 \mathrm{~min}$. An $\sim 50 \mathrm{~nm}$ compact $\mathrm{TiO}_{2}$ layer was deposited by spincoating a clear solution of $\mathrm{TiO}_{2}$ at $3000 \mathrm{rpm}$ for $20 \mathrm{~s}$; and then annealing at $450{ }^{\circ} \mathrm{C}$ for $30 \mathrm{~min}$. The $\mathrm{TiO}_{2}$ solution was synthesized by a sol-gel method from a precursor prepared by mixing $5 \mathrm{~mL}$ of EtOH, two drops of $\mathrm{HCl}, 125 \mathrm{~mL}$ of deionized water, and $375 \mathrm{~mL}$ of titanium ethoxide. The QD absorber layer was deposited following the procedure described above. Spiro-OMeTAD films were deposited by spin-coating at $5000 \mathrm{rpm}$ for $30 \mathrm{~s}$ from a solution containing 72.3 $\mathrm{mg}$ spiro-OMeTAD, 28.8 $\mu \mathrm{L} 4$-TBP and $17.5 \mu \mathrm{L}$ of a bis(trifluoromethane)sulfonimide lithium salt (LiTFSI) stock solution $\left(520 \mathrm{mg} \mathrm{mL}^{-1}\right.$ in acetonitrile) dissolved in $1 \mathrm{~mL}$ of chlorobenzene. All the spin-coating processes were carried out under ambient conditions unless otherwise specified. $\mathrm{MoO}_{x}$ was evaporated at a rate of 0.1 to $0.5 \AA \mathrm{s}^{-1}$ at a base pressure lower than $2 \times 10^{-7}$ torr, resulting in a total thickness of $15 \mathrm{~nm}$. Al electrodes were deposited at a rate of 0.5 to 2.0 $\AA \mathrm{s}^{-1}$ for a total thickness of $200 \mathrm{~nm}$. The encapsulation of devices was 
processed in a $\mathrm{N}_{2}$-filled glovebox by sealing the active area to another piece of glass with polyisobutylene tape.

Characterization of QD solutions and films. TEM images were obtained using a FEI Tecnai F20 electron microscope with $200 \mathrm{kV}$ accelerating voltage. To determine elemental and isotopic distributions in solids, as well as the structure and composition of organic materials, time-of-flight secondary ion mass spectrometry (ToF-SIMS) is a powerful analytical technique to investigate HPSC materials and devices ${ }^{13,27}$. An ION-ToF ToF-SIMS V spectrometer was utilized to depth-profile the $\mathrm{Cs}_{1-x} \mathrm{FA}_{x} \mathrm{PbI}_{3}$ QD films. Analysis was carried out utilizing a 3-lens $30 \mathrm{kV}$ BiMn primary ion gun, and the $\mathrm{Bi}_{3}+$ primary-ion beam (operated in bunched mode; 21 ns pulse width, analysis current $0.7 \mathrm{pA}$ ) was scanned over a $25 \times 25$ micron area. Depth Profiling was completed with a $1 \mathrm{kV}$ oxygen ion sputter beam $(7 \mathrm{nA}$ sputter current) rastered over a $150 \times 150$ micron area. All spectra during profiling were recorded at or below a primary ion dose density of $1 \times 10^{12}$ ions $\mathrm{cm}^{-2}$ to remain at the static-sims limit.

Transient absorption spectra (TA) were recorded using a Continuum Integra-C laser, with an output of $800 \mathrm{~nm}$ at $1 \mathrm{kHz}$. The $800 \mathrm{~nm}$ beam was directed into a Palitra optical parametric amplifier to generate pump pulse (about $150 \mathrm{fs}$ ) at $400 \mathrm{~nm}$ and was modulated at $500 \mathrm{~Hz}$ through an optical chopper to block every other laser pulse. Femtosecond TA spectra were collected using Helios spectrometer (Ultrafast Systems). A small amount of $800 \mathrm{~nm}$ light was used to pump a sapphire crystal to create $450-800 \mathrm{~nm}$ probe light for TA.

For time-resolved microwave conductivity (TRMC) measurements, the perovskite QD films deposited onto pre-cleaned quartz substrates $(1 \mathrm{~cm} \times 2.5 \mathrm{~cm} \times$ $1 \mathrm{~mm}$ ) were photoexcited through the quartz side of the substrate with $650 \mathrm{~nm}(5$ ns pulse width) pules at $10 \mathrm{~Hz}$ from an optical parametric oscillator (Continuum Panther) pumped by the $355 \mathrm{~nm}$ harmonic of a Q-switched Nd:YAG laser (Continuum Powerlite). The transient change in photoconductance, $\Delta G(t)$, was recorded via transient change in the microwave power, $\Delta P(t)$, due to absorption of microwaves (ca. $9 \mathrm{GHz}$ ) by mobile photogenerated charge carriers in the sample. $\Delta G(t)$ was calculated by:

$$
\Delta G(t)=\left(-\frac{1}{K}\right)\left(\frac{\Delta P(t)}{P}\right)
$$

where $K$ is a calibration factor experimentally determined from the dielectric properties of the QD films and the resonance characteristics of the microwave cavity. The end-of-pulse (peak) photoconductance, $\Delta G_{\text {peak }}$, is related to the yield mobility product $(\varphi \Sigma \mu)$ using the equation:

$$
\Delta G_{\text {peak }}=\beta q_{\mathrm{e}} N\left(\mu_{\mathrm{e}}+\mu_{\mathrm{h}}\right)=\beta q_{\mathrm{e}} I_{0} F_{\mathrm{A}} \varphi \sum \mu
$$

where $\beta=2.2$ and is the ratio of the interior dimensions of the waveguide, $\varphi$ is the yield of free-carrier generation, $q_{\mathrm{e}}$ is the electronic charge, $N$ is the number of photogenerated charge-carrier pairs, $\mu_{\mathrm{e}}$ and $\mu_{\mathrm{h}}$ are the electron and hole mobilities (termed $\Sigma \mu), I_{0}$ is the incident photon flux of the excitation laser pulse, and $F_{\mathrm{A}}$ is the fraction of photons $(650 \mathrm{~nm})$ absorbed by the QD films. Pump excitation intensities were decreased until peak photoconductivity values and transient lifetime were intensity independent, provided sufficient signal-to-noise. At $650 \mathrm{~nm}$, generation of charges can be uniform throughout the vertical cross section of the films, thus further mitigating higher-order recombination processes. Hence, we extracted the carrier mobilities of the QD films from this linear response region of the data.

XPS and UPS measurements were performed on a Kratos Axis Nova instrument, using monochromated $\mathrm{Al}-\mathrm{K} \alpha(\mathrm{hv}=1486.7 \mathrm{eV})$, and $\mathrm{He}-\mathrm{I}(\mathrm{hv}=21.22$ $\mathrm{eV}$ ) excitation sources, respectively. The spectrometer binding-energy (BE) scale was calibrated by measuring valence-band and core-level spectra from sputtercleaned $\mathrm{Au}$ and $\mathrm{Cu}$ foils $\left(E_{\mathrm{F}}=0.00 \mathrm{eV}, \mathrm{Au} 4 f_{7 / 2}=83.96 \mathrm{eV}\right.$, and $\mathrm{Cu} 2 p_{3 / 2}=932.62$ $\mathrm{eV})^{28}$. XPS/UPS spectra were acquired at pass energy $E_{\text {pass }}=20 \mathrm{eV}$. The reference energy levels for spiro-OMeTAD and $\mathrm{TiO}_{2}$ were taken from literature ${ }^{29}$

Characterization of PV devices. Devices were tested on a Newport Oriel Sol3A solar simulator with xenon lamp in the glove box at room temperature. The intensity of the solar simulator was calibrated to $100 \mathrm{~mW} \mathrm{~cm}^{-2}$ AM1.5 G with a KG5 filtered Si reference solar cell that was certified by NREL PV Performance Characterization Team and the spectral mismatch factor was minimized to 0.9923 . $J-V$ scans were measured from forward bias to reverse bias step and from reverse bias to forward (bias size: $100 \mathrm{mV}$, delay time: $10 \mathrm{~ms}$, number of power line cycles: 0.1 , respectively. The devices were masked with a black metal aperture to define an active area of $0.059 \mathrm{~cm}^{2}$. The stabilized power output (SPO) of devices was measured by holding the illuminated devices at a constant voltage near the maximum power point of the $J-V$ scan and recording the continuous current output in the meantime. External quantum efficiency (EQE) measurements were performed utilizing a Newport Oriel IQE200.

To obtain the cross-sectional TEM images of devices, a Pt capping layer was deposited on the surface of devices to protect the thin-film structure. TEM foils were prepared by the standard focused ion beam (FIB) lift-out technique and then the final thickness was reduced to less than $<100 \mathrm{~nm}$ by a Ga ion milling. To remove preparation damage on the TEM foils, a Fischione Nanomill was used and a low energy cleaning was carried out at $500 \mathrm{eV}$ under vacuum of $10^{-7}$ torr at $-170^{\circ} \mathrm{C}$ for $\pm 10^{\circ}$. TEM observations were operated on a FEI Tecnai F20 TEM at an acceleration voltage of $200 \mathrm{kV}$. Chemical analysis was performed with energydispersive X-ray spectrometry (EDS) in the high-angle annular dark-field (HAADF)-scanning TEM (STEM) mode.

\section{Data availability}

Data that support the plots within this work are available from the corresponding author upon reasonable request.

Received: 19 February 2019 Accepted: 20 May 2019

Published online: 28 June 2019

\section{References}

1. Ning, Z. et al. Graded doping for enhanced colloidal quantum dot photovoltaics. Adv. Mater. 25, 1719-1723 (2013).

2. Haschke, J., Dupré, O., Boccard, M. \& Ballif, C. Silicon heterojunction solar cells: recent technological development and practical aspects - from lab to industry. Sol. Energy Mater. Sol. Cells 187, 140-153 (2018).

3. Zhao, T. et al. Advanced architecture for colloidal PbS quantum dot solar cells exploiting a CdSe quantum dot buffer layer. ACS Nano 10, 9267-9273 (2016).

4. Adinolfi, V. et al. Electric field engineering using quantum-size-effect-tuned heterojunctions. Appl. Phys. Lett. 103, 011106 (2013).

5. Crisp, R. W. et al. Metal halide solid-state surface treatment for high efficiency PbS and PbSe QD solar cells. Sci. Rep. 5, 9945 (2015).

6. Etgar, L., Gao, P., Qin, P., Graetzel, M. \& Nazeeruddin, M. K. A hybrid lead iodide perovskite and lead sulfide QD heterojunction solar cell to obtain a panchromatic response. J. Mater. Chem. A 2, 11586-11590 (2014).

7. Luque, A. L. \& Viacheslav, A. in Concentrator Photovoltaics (eds Luque, A. L. \& Viacheslav, A.) 25-50 (Springer, Berlin, Heidelberg, 2007).

8. Jiang, C.-S. et al. Direct evidence of a buried homojunction in $\mathrm{Cu}(\mathrm{In}, \mathrm{Ga}) \mathrm{Se}_{2}$ solar cells. Appl. Phys. Lett. 82, 127-129 (2002).

9. Strohm, A. et al. $\mathrm{ZnO} / \mathrm{InxSy} / \mathrm{Cu}(\mathrm{In}, \mathrm{Ga}) \mathrm{Se}_{2}$ solar cells fabricated by coherent heterojunction formation. Thin Solid Films 480-481, 162-167 (2005).

10. Sanehira, E. M. et al. Enhanced mobility $\mathrm{CsPbI}_{3}$ quantum dot arrays for record-efficiency, high-voltage photovoltaic cells. Sci. Adv. 3, eaao4204 (2017)

11. Swarnkar, A. et al. Quantum dot-induced phase stabilization of a-CsPbI perovskite for high-efficiency photovoltaics. Science 354, 92-95 (2016).

12. Wheeler, L. M. et al. Targeted ligand-exchange chemistry on cesium lead halide perovskite quantum dots for high-efficiency photovoltaics. J. Am. Chem. Soc. 140, 10504-10513 (2018).

13. Harvey, S. P. et al. Probing perovskite inhomogeneity beyond the surface: TOF-SIMS analysis of halide perovskite photovoltaic devices. ACS Appl. Mater. Interfaces 10, 28541-28552 (2018).

14. Zolfaghari, Z. et al. Operation mechanism of perovskite quantum dot solar cells probed by impedance spectroscopy. ACS Energy Lett. 4, 251-258 (2019)

15. Bian, $\mathrm{H}$. et al. Graded bandgap $\mathrm{CsPbI} \mathrm{Pl}_{2+\mathrm{x}} \mathrm{Br}_{1-\mathrm{x}}$ perovskite solar cells with a stabilized efficiency of $14.4 \%$. Joule 2, 1500-1510 (2018).

16. Moriarty, T. \& Levi, D. in Proc. 2017 IEEE 44th Photovoltaic Specialist Conference (PVSC) 483-486 (IEEE, Washington, DC, USA, 2017).

17. Zimmermann, E. et al. Characterization of perovskite solar cells: Towards a reliable measurement protocol. APL Mater. 4, 091901 (2016).

18. Park, S. H. et al. Bulk heterojunction solar cells with internal quantum efficiency approaching 100\%. Nat. Photon. 3, 297 (2009).

19. Chen, X., Lu, H., Yang, Y. \& Beard, M. C. Excitonic effects in methylammonium lead halide perovskites. J. Phys. Chem. Lett. 9, 2595-2603 (2018).

20. Calik, M. et al. Extraction of photogenerated electrons and holes from a covalent organic framework integrated heterojunction. J. Am. Chem. Soc. 136, 17802-17807 (2014)

21. Lan, X., Masala, S. \& Sargent, E. H. Charge-extraction strategies for colloidal quantum dot photovoltaics. Nat. Mater. 13, 233 (2014).

22. $\mathrm{Wu}, \mathrm{K}$. et al. Ultrafast interfacial electron and hole transfer from $\mathrm{CsPbBr}_{3}$ perovskite quantum dots. J. Am. Chem. Soc. 137, 12792-12795 (2015)

23. $\mathrm{Hu}, \mathrm{Y}$. et al. Hybrid perovskite/perovskite heterojunction solar cells. ACS Nano. 10, 5999-6007 (2016).

24. Cui, P. et al. Planar $\mathrm{p}-\mathrm{n}$ homojunction perovskite solar cells with efficiency exceeding 21.3\%. Nat. Energy 4, 150-159 (2019).

25. Protesescu, L. et al. Nanocrystals of cesium lead halide perovskites $\left(\mathrm{CsPbX}_{3}\right.$ $\mathrm{X}=\mathrm{Cl}, \mathrm{Br}$, and I): novel optoelectronic materials showing bright emission with wide color gamut. Nano Lett. 15, 3692-3696 (2015).

26. Hazarika, A. et al. Perovskite quantum dot photovoltaic materials beyond the reach of thin films: full-range tuning of A-site cation composition. ACS Nano. 12, 10327-10337 (2018)

27. Christians, J. A. et al. Tailored interfaces of unencapsulated perovskite solar cells for $>1,000$ h operational stability. Nat. Energy 3, 68-74 (2018). 
28. Seah, M. P., Gilmore, I. S. \& Beamson, G. XPS: binding energy calibration of electron spectrometers $5-$ re-evaluation of the reference energies. Surf. Interface Anal. 26, 642-649 (1998).

29. Jiménez-López, J., Cambarau, W., Cabau, L. \& Palomares, E. Charge injection, carriers recombination and HOMO energy level relationship in perovskite solar cells. Sci. Rep. 7, 6101 (2017).

\section{Acknowledgements}

This work was authored by Alliance for Sustainable Energy, LLC, the manager and operator of the National Renewable Energy Laboratory for the U.S. Department of Energy (DOE) under contract no. DE-AC36-08GO28308. We acknowledge the Department of Defense Operational Energy Capability Improvement Fund (OECIF) for development of these QD solar cells. Transient absorption work is supported from the Center for Hybrid Organic Inorganic Semiconductors for Energy (CHOISE) an Energy Frontier Research Center funded by the Office of Basic Energy Sciences, Office of Science within the U.S. Department of Energy. Q.Z. acknowledges fellowship support from the China Scholarship Council and Natural Science of Foundation China (21576140). The views expressed in the article do not necessarily represent the views of the DOE or the U. S. Government.

\section{Author contributions}

Q.Z. and J.M.L. conceived the project, analyzed all data, and wrote the first draft. Q.Z. fabricated the devices and prepared samples for measurements. Q.Z. prepared quantum dots under the guidance of A.H. X.C. constructed the TA experimental set-up and performed the TA measurements. M.C.B. analyzed TA data. S.P.H. performed ToFSIMS measurements and analyzed the data. G.R.T. carried out XPS and UPS measurements and film energetics simulation and analyzed the data. B.W.L. performed TRMC measurements. J.L. and C.X. performed imaging measurements. Q.Z. encapsulated devices under the guidance of T.S. and L.S. J.M.L. supervised the entire project. G.L. and M.Z. discussed all results. All authors contributed to the final writing of the manuscript.

\section{Additional information}

Supplementary Information accompanies this paper at https://doi.org/10.1038/s41467019-10856-z.

Competing interests: The authors declare no competing interests.

Reprints and permission information is available online at http://npg.nature.com/ reprintsandpermissions/

Peer review information: Nature Communications thanks Weijie Zhao and other anonymous reviewer(s) for their contribution to the peer review of this work.

Publisher's note: Springer Nature remains neutral with regard to jurisdictional claims in published maps and institutional affiliations.

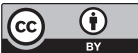

Open Access This article is licensed under a Creative Common Attribution 4.0 International License, which permits use, sharing, adaptation, distribution and reproduction in any medium or format, as long as you give appropriate credit to the original author(s) and the source, provide a link to the Creative Commons license, and indicate if changes were made. The images or other third party material in this article are included in the article's Creative Commons license, unless indicated otherwise in a credit line to the material. If material is not included in the article's Creative Commons license and your intended use is not permitted by statutory regulation or exceeds the permitted use, you will need to obtain permission directly from the copyright holder. To view a copy of this license, visit http://creativecommons.org/ licenses/by/4.0/.

(C) The Author(s) 2019 